\title{
Perspective
}

PERSPECTIVE Actualité en histoire de l'art

2| 2007

La Grande-Bretagne/Période moderne

\section{Le culte des images, le désir des sorcières. Travaux récents sur l'art allemand de la Renaissance}

\section{François-René Martin}

\section{(2) OpenEdition}

1 Journals

Édition électronique

URL : http://journals.openedition.org/perspective/3831

DOI : $10.4000 /$ perspective.3831

ISSN : 2269-7721

Éditeur

Institut national d'histoire de l'art

Édition imprimée

Date de publication : 30 juin 2007

Pagination : 377-381

ISSN : 1777-7852

\section{Référence électronique}

François-René Martin, « Le culte des images, le désir des sorcières. Travaux récents sur l'art allemand de la Renaissance », Perspective [En ligne], 2 | 2007, mis en ligne le 31 mars 2018, consulté le 01 octobre 2020. URL : http://journals.openedition.org/perspective/3831; DOI : https://doi.org/10.4000/ perspective.3831 


\title{
Le culte des images, le désir des sorcières. Travaux récents sur l'art allemand de la Renaissance
}

\author{
François-René Martin
}

\section{RÉFÉRENCE}

Edgar Bierende, Lucas Cranach d. Ä. und der deutsche Humanismus. Tafelmalerei im Kontext von Rhetorik, Chroniken und Fürstenspiegeln, Munich/Berlin, Deutscher Kunstverlag, 2002, 518 p., 110 fig. n. et b. ISBN : 978-3-422-06339-6 ; $68 €$.

Bodo Brinkmann, Hexenlust und Südenfall. Die Seltsamen Phantasien des Hans Baldung Grien. Witches' Lust and the Fall of Man. The Strange Phantasies of Hans Baldung Grien (avec un essai de Berthold Hinz), Francfort-s.-M./Petersberg, Städel Museum/Michael Imhof, 2006. 272 p., 156 fig. coul. ISBN : 978-3-86568-225-3 ; 29,95€.

Magdalena Bushart, Sehen und Erkennen. Albrecht Altdorfers religiöse Bilder, Munich/ Berlin, Deutscher Kunstverlag, 2004. 365 p., 239 fig. n. et b. et coul. ISBN :

978-3-422-06455-3; $88 €$.

Thomas Noll, Albrecht Altdorfer in seiner Zeit. Religiöse und profane Themen in der Kunst um 1500, Munich/Berlin, Deutscher Kunstverlag, 2004.582 p., 202 fig. n. et b. ISBN :

978-3-422-06454-6; $98 €$.

Charles Zika, Exorcising Our Demons. Magic, Witchcraft and Visual Culture in Early Modern Europe, Leyde, Brill, 2003.610 p., env. 120 fig. n. et b. ISBN ; 978-90-04-12560-5 ; $99 €$.

1 En l'espace de quelques années, la connaissance que nous avons de l'art de quelquesuns des plus grands artistes de la fin du Moyen Âge et de la Renaissance allemande a été considérablement renouvelée. L'art de Martin Schongauer, par exemple, ou d'un de ses prédécesseurs les plus éminents, le Strasbourgeois Jost Haller, ont été récemment reconsidérés, l'art du Rhin supérieur ayant fait l'objet d'une très vaste présentation à Karlsruhe à la fin de l'année 2001'. Après Stefan Lochner, l'œuvre du Maître du Retable de Saint Barthélemy fut révélée dans un vaste contexte stylistique, entre Memling et la 
sculpture à Utrecht à la fin du $\mathrm{xv}^{\mathrm{e}}$ siècle, à l'occasion d'une exposition au WallrafRichartz Museum à Cologne, en 2001². Dürer, Holbein et Grünewald continuent à faire l'objet d'une littérature relativement abondante, les expositions jouant là encore leur rôle dans la diffusion des dernières recherches ${ }^{3}$. Il faut cependant mentionner des études fondamentales comme celle que Katherine Crawford Luber a consacrée au rapport entre Dürer et la Renaissance vénitienne. Question des plus sensibles dans l'historiographie allemande et dans laquelle l'auteur s'immisce en venant contester, avec les arguments les plus forts, l'hypothèse d'un voyage en Italie en 1494, hypothèse à laquelle croyaient des esprits aussi peu vulnérables aux conjectures fragiles que Moritz Thausing, Heinrich Wölfflin ou encore Erwin Panofsky

\section{Amour sacré}

2 Nombre de ces travaux récents ont en commun de tenter de livrer de nouvelles interprétations des œuvres de ces artistes en les rapportant à leur environnement culturel immédiat - en l'occurrence l'univers des théologiens et des humanistes entourant et déterminant une part de la signification de leurs créations. L'enquête récente de David Hotchkiss Price sur Dürer, qui éclaire des œuvres aussi insignes que l' Apocalypse (1498) ou les Quatre apôtres (1526) en les reliant aux idées véhiculées par Pirckheimer et son cercle, à la littérature joachimite ou aux thèses luthériennes en est un exemple ${ }^{5}$. Cranach est évidemment l'artiste pour lequel le problème des relations avec l'univers des théologiens est le plus débattu. Dans le prolongement des travaux d'Andreas Tacke et parallèlement à ceux de Sabine Heiser ${ }^{6}$, le livre d'Edgar Bierende, Lucas Cranach d. Ä. und der deutsche Humanismus, montre l'artiste à la découverte du discours des humanistes, des traités de rhétoriques ou des chroniques sur l'Antiquité germanique. Les longs développements que l'auteur consacre au rôle supposé de la sculpture dans l'imaginaire historiographique de Cranach, tout particulièrement le monument funéraire de Frédéric III de Nicolaus Gerhaert de Leyde (1467-1473) à Vienne, lui permettent de montrer combien, dans cette version allemande d'un retour à l'antique, la plongée vers les origines engageait également des valeurs formelles jadis exprimées dans la sculpture par Gerhaert. C'est le même imaginaire historiographique que l'on retrouve dans les peintures mythologiques de Cranach, comme Vénus et Cupidon (1509) ou encore la Nymphe à la source (1518), indissociables du sanctuaire de Diane à Magdebourg ou de la source de Meissen, alors vénérés dans le milieu humaniste.

Que la rhétorique, avec l'imaginaire dynastique et historiographique, soit un des horizons de l'art allemand de la Renaissance (et par la même une des clés d'interprétation de nombre d'œuvres de Cranach ou de Dürer), l'ouvrage de Bierende en fait la démonstration, se fondant notamment sur un célèbre passage de Melanchton (Elementorum rhetorices libri duo, 1531), selon qui l'art de Dürer relevait du genus grande, celui de Cranach du genus humile, alors que Matthias (Grünewald) occupait le milieu, le genus mediocre ${ }^{7}$. Melanchton posait en réalité la question du pouvoir de révélation du divin propre à la peinture qui s'affirme alors à la fois comme un modèle efficace de communication de la vérité théologique et une source pluraliste pour la Réforme allemande. Ce pouvoir de communication théologique propre à la peinture est au centre de deux autres livres récemment publiés et consacrés à un autre grand artiste de la Renaissance auquel Melanchton ne faisait pas allusion : Albrecht Altdorfer. Alors que 
Thomas Noll (Albrecht Altdorfer in seiner Zeit...) accorde la plus grande importance aux questions de modification des schèmes iconographiques, Magdalena Bushart (Sehen und Erkennen...) fait du spectateur l'instance délivrant leur sens aux complexes montages iconographiques de l'artiste. Qu'il s'agisse du problème de l'amour divin et de l'amour profane (Noll), de figuration de l'amour pour le Christ ou de Concupiscentia oculorum (Bushart), l'un et l'autre ont cependant en commun d'examiner l'imagerie religieuse d'Altdorfer en la mettant en miroir de son imagerie profane. L'un et l'autre, enfin, situent la production d'Altdorfer dans un contexte de crise de l'image cultuelle et d'individualisation de la création artistique.

\section{Amour profane}

Dans les constellations d'images illustrant le désir apparaissent inévitablement des exemples empruntés à Hans Baldung Grien, qui posent de redoutables problèmes d'interprétation. C'est tout l'intérêt de l'essai de Bodo Brinkmann qui accompagne l'exposition récemment consacrée aux sorcières chez Baldung au Städel Museum à Francfort, que de reprendre le dossier, dans un essai à la fois rigoureux et original. Le point de départ est le célèbre panneau représentant Deux sorcières (cat. 1). Jadis en possession de Giovanni Morelli, l'œuvre fut acquise par le Städel en 1878, comme un tableau authentique de l'artiste (le monogramme désignant sans ambiguïté l'auteur) représentant une allégorie de l'amour sacré et de l'amour profane. Ce n'est qu'autour de 1900 que les historiens de l'art s'orientèrent vers les croyances populaires et les superstitions et virent dans les deux femmes représentées des sorcières. Baldung avait peint cette œuvre à un moment où, dans le Saint Empire germanique, des femmes avaient été incriminées, condamnées, torturées et mises à mort comme sorcières. Aussi les interprètes de l'œuvre cherchèrent-ils à rapporter l'action représentée aux catégories criminelles en usage : les deux femmes seraient ainsi en train de produire un orage de grêle ou une tornade, ce qui en ferait en quelque sorte des «sorcières météorologiques » œuvrant aux intempéries. Les traités de droit de l'époque abondent en gravures représentant des sorcières jetant dans des marmites des serpents ou des volatiles et provoquant instantanément des orages: le De Laminis d'Ulrich Monito (publié à Ulm en 1489), ou le Layenspiegel d'Ulrich Tengler (Augsburg, 1511) donnent à voir de telles scènes. Il reste que le tableau de Francfort, comme le montre avec la plus grande clarté Brinkmann, ne représente en aucune façon une telle opération de sorcellerie. Aucune cuisine maléfique, et encore moins de vol nocturne: le bouc est littéralement écrasé par la sorcière de droite et aveuglé par le voile. Quant à la tempête, il ne s'agit pas de grêle mais de feu, et n'a pas de causalité avec l'attitude des sorcières ni même du putto. Brinkmann met en relation cette disjonction avec l'attitude libérale à l'égard des sorcières du grand prédicateur Johann Geiler von Kaisersberg, un des théologiens les plus en vue à Strasbourg autour de 1500, pour qui le vol des sorcières était en réalité une suggestion diabolique. Le vol était dans la conscience de ces femmes; le diable, entendant les signes et les paroles des sorcières, agit à leur place. Aussi ces pratiques n'étaient-elles jamais que des signes et non des actes efficaces.

5 En cela, la représentation des sorcières, chez Baldung, n'illustre en aucune façon de manière littérale les représentations ordinaires de la sorcellerie. Pour ne prendre qu'un exemple qui est au centre des travaux de Charles Zika sur la sorcellerie (Exorcising our Demons...), les sorcières de Francfort ou les dessins de l'artiste représentant des scènes 
de sabbat sont à l'opposé des Mélancolies d'un Cranach, dénonçant les illusions créées par le désir en associant les hordes sauvages passant dans les airs au désordre sexuel ${ }^{8}$. Les sorcières de Baldung ont été conçues pour des lecteurs de Geiler, aptes à saisir la dimension humoristique d'une telle œuvre, à discourir de la causalité des choses et de la suggestion diabolique, mais aussi à admirer le teint d'ivoire des corps des deux sorcières. Celles-ci sont en réalité les muses de ces humanistes, en même temps que les modèles dénudés de Baldung 9 , en bref des " sorcières artistiques » comme l'écrit de son côté Brinkmann. Le lieu où une telle œuvre était destinée, où elle trouvait naturellement son public, est très probablement la Kunstkammer, le cabinet de curiosité. Ignorant les caractérisations juridiques et théologiques en usage au sujet des sorcières, jouant d'un point de vue formel sur les équivalences avec la sculpture (celles, par exemple, de Conrad Meit ou de Thomas Hering), l'œuvre de Baldung défiait les catégories, transcendait les limites entre les médiums artistiques. Quel public, sinon celui des Geiler, Sebastian Brant ou Beatus Rhenanus était alors en mesure de percevoir que l'œuvre n'avait pas été conçue d'un point de vue narratif mais discursif? $\mathrm{Ne}$ racontant pas une histoire, n'impliquant pas une signification morale ou allégorique, les Deux sorcières de Francfort convoient tout une chaîne d'associations. Le message parle ainsi de différentes manières à plusieurs spectateurs, rendant inévitable la discussion. C'est l'ambiguïté profonde de l'œuvre qui fonde sa discursivité.

\section{Le démon de l'interprétation}

6 Le discours des humanistes sur ces sorcières de Baldung pouvait ainsi s'étendre à l'infini. Mais la faible idée que nous avons de ce milieu et des cercles que fréquentait Baldung ne nous permet que difficilement d'articuler fermement à un public précis les significations projetées sur ses cuvres $^{10}$. C'est ce qui fonde alors le travail d'interprétation de l'historien de l'art, en quête de textes ou de références à d'autres œuvres contemporains. Or la dimension réflexive de l'art de Baldung est patente. Il procède à un très haut degré d'une méditation personnelle sur l'art des autres artistes, et en premier lieu d'un long et minutieux examen critique de l'art de Dürer. Ainsi, dans son rapport avec l'art de ce dernier ou de ses contemporains, Grünewald spécialement, la création chez Baldung apparaît-elle comme un intense effort de déplacement des formules, de recodification des significations et d'intensification de la participation du spectateur, "Kunst über Kunst» comme l'écrit Brinkmann. C'est ce qui autorise ce dernier à déboîter de petits paragraphes, comme autant de commentaires gigognes, sur nombre de questions que les Deux sorcières sont susceptibles de poser : l'utilisation des schémas des cartes à jouer et des marges enluminées ; la reprise de la question du nu et des proportions telle que Dürer avait tenté de la codifier; celle, brûlante, de la connaissance par Baldung du dialogue Erotes de Lucien de Samosate ; la vision du sexe féminin ; le thème de la prostitution et de la syphilis, auquel la fiole que tient une des deux sorcières de Francfort ferait directement allusion; enfin celui de la Chute et du péché originel, omniprésents dans les dernières compositions de l'artiste et dont $L a$ Mort embrassant une femme, de Bâle, serait une des formulations les plus saisissantes.

7 Reste la dimension auto-référentielle dans l'art de Baldung qui est tout aussi importante et qui irriguait le commentaire aussi fascinant que discutable de Joseph Leo Koerner, dans The Moment of Self-Portraiture in German Renaissance Art ${ }^{11}$. Si Brinkmann, qui voit dans les sorcières de Francfort tout comme dans la plupart des œuvres qu'il 
leur rattache une confession personnelle dans laquelle l'artiste formule ses vues sur la sexualité humaine, ne conteste pas l'essentiel de l'analyse de Koerner sur la série gravée des chevaux sauvages, il reste plus circonspect au sujet de la fameuse gravure dite du Palefrenier ensorcelé. Là où Koerner, dans des pages emphatiques qui faisaient suite à celles de Gustav Hartlaub, voyait un autoportrait de l'artiste et plus encore une véritable prise de position théorique sur la "crise de l'interprétation " trouvant son achèvement dans la mort de l'artiste, Brinkmann ne voit guère dans cette gravure cryptée que les songes d'un " homme ordinaire ", assertion explicitée dans un excursus placé à la fin de l'ouvrage sur l'affinité entre l'obsession de Baldung pour la sexualité et l'œuvre de l'écrivain français Michel Houellebeck. Ce détour par la littérature contemporaine, qui a la vertu de donner aux œuvres de Baldung toute leur radicalité, nous fait regretter en réalité qu'une véritable explication avec les thèses de Koerner n'ait pu trouver sa place dans cet essai. En se démarquant des travaux essentiels de Jean Wirth, qui avait tenté de localiser la signification de quelques-unes des allégories macabres de Baldung (La Mort et la Femme; Eve, le serpent et la femme; La Musique et la Prudence; Les Trois grâces et Les Trois âges de la vie) en les rapprochant de textes d'Agrippa de Netesheim, de Paracelse et surtout de l'influent théoricien du nicodémisme, Brunfels ${ }^{12}$, Koerner entendait éloigner la tâche de l'historien de toute recherche vaine d'une doctrine ou d'un texte qui les stabiliserait et les renverrait à un spectateur historiquement situé ${ }^{13}$. La référence à Paul de Man planait du reste sur les analyses hautement spéculatives de Koerner, à rebours de l'esthétique de la réception, et qui faisaient de la démarche autobiographique un acte de défiguration (defacement). L'art de Baldung, plus encore que celui de Dürer, était absorbé dans ce penchant déictique et autobiographique, qui voyait alors dans la mort le thème le plus apte à permettre aux significations de proliférer dans les œuvres de Baldung et en même temps de les occulter, rendant ainsi la tâche de l'interprète interminable ${ }^{14}$. L'histoire de l'art n'en a pas fini avec Baldung, ni même avec ses interprètes.

\section{NOTES}

1. Stephan Kemperdick, Martin Schongauer - eine Monographie, Petersberg, 2004 ; Philippe Lorentz, Jost Haller: le peintre des chevaliers et l'art en Alsace au XV siècle, (cat. expo., Colmar, Musée d'Unterlinden, 2001), Paris/Colmar, 2001; Spätmittelalter am Oberrhein. Maler und Werkstätten, 1450-1525, (cat. expo., Staatliche Kunsthalle Karlsruhe, 2001), Stuttgart, 2001.

2. Rainer Budde, Roland Krischel éd., Genie ohne Namen. Der Meister des Bartholomäus-Altars, (cat. expo., Cologne, Wallraf-Richartz Museum, 2001), Cologne, 2001. Voir également Frank Günter Zehnder éd., Stefan Lochner Meister zu Köln. Herkunft - Werke - Wirkung, (Cat. expo., Cologne, Wallraf-Richartz Museum, 1993-1994), Cologne, 1993.

3. Giulia Bartrum éd., Albrecht Dürer and his Legacy. The Graphic Work of a Renaissance Artist, (cat. expo., Londres, British Museum, 2002), Londres, 2002 ; Susan Foister, Dürer and the Virgin in the Garden, (cat. expo., Londres, National Gallery, 2004), New Haven/Londres, 2004 ; Der Bürgermeister, sein Maler une seine Familie. Hans Holbeins Madonna im Städel, (cat. expo., Francfort-s.-M., Städel Museum, 2004), Petersberg, 2004 ; Hans Holbein the Younger. The Basel Years 1515-1532, (cat. expo., 
Bâle, Kunstmuseum, 2006), Munich/Berlin/Londres/New York, 2006 ; Rainhard Riepertinger, Evamaria Brockhoff, Katharina Heinemann, Jutta Schumann éd., Das Rätsel Grünewald, (cat. expo., Aschaffenburg, Schloss Johanissburg/Augsburg, Haus der Bayerischen Geschichte, 2002), Augsburg, 2002. Au sujet de ce dernier artiste, on signalera la préparation de deux expositions conjointes qui ouvriront à la fin de l'année 2007 : Grünewald. Regards sur un chef-d'œuvre, Colmar, Musée d'Unterlinden; Grünewald et son temps, Staatliche Kunsthalle Karlsruhe. Les musées de Berlin préparent eux aussi une exposition sur Grünewald pour 2008.

4. Katherine Crawford Luber, Albrecht Dürer and the Venetian Renaissance, Cambridge, 2005.

5. David Hotchkiss Price, Albrecht Dürer's Renaissance: Humanism, Reformation, and the Art of Faith, Ann Arbor, 2003.

6. Andreas Tacke, Der katolische Cranach. Zu zwei Grossauträgen von Lucas Cranach d.Ä., Simon Franck und der Cranach-Werkstatt (1520-1540), (Berliner Schriften zur Kunst, 2), Mayence, 1992 ; Sabine Heiser, Das Frühwerk Lucas Cranachs des Älteren. Wien und Dresden um 1900, Berlin, 2002.

7. Voir à ce sujet BIERENDE, Lucas Cranach, p. 52.

8. Sur les problèmes d'interprétation de cette série, voir outre Zika: Hanne Kolind Poulsen, "Choice and Redemption. On Lucas Cranach the Elder's Melancholia in Statens Museum for Kunst ", dans Statens Museum for Kunst Journal, Copenhague, 4, 2000, p. 41-75.

9. Comme vient de le soutenir Linda C. Hult, The Witch as Muse. Art, Gender and Power in Early Modern Europe, Philadelphie, 2005.

10. En dépit des efforts de Thomas A. Brady, «The Social Place of German Renaissance Artist: Hans Baldung Grien (1484/5-1545) », dans Central European History, 8, 1975, p. 295-315.

11. Joseph Leo Koerner, The Moment of Self-Portraiture in German Renaissance Art, Chicago, 1993, notamment p. 455 et 470.

12. Jean Wirth, La Jeune fille et la mort. Recherches sur les thèmes macabres dans l'art germanique de la Renaissance, Genève, 1979, particulièrement p. 150 et suivantes. Brinkmann redonne d'ailleurs vigueur aux rapprochements faits par Wirth avec les thèses d'Agrippa de Nettesheim.

13. Voir J.L. Koerner, «The Mortification of the Image: Death as a Hermeneutic in Hans Baldung Grien », dans Representations, 10, 1985, p. 52-101, p. 93.

14. Voir à ce sujet «Wanderjahre. Ein Interview von Pamela Lee mit Joseph Leo Koerner », dans Texte zur Kunst, 4/14, juin 1994, p. 114-123 et dans le même numéro Christopher Wood, « Paul de Man und die Kunstgeschichte », p. 83-89.

\section{INDEX}

Keywords : german art, art history, historiography, humanism, image, witchcraft, iconography, interpretations, woman

Index géographique : Allemagne

Mots-clés : art allemand, histoire de l'art, historiographie, humanisme, image, sorcellerie, iconographie, interprétations, femme

Index chronologique : 1500 


\section{AUTEURS}

\section{FRANÇOIS-RENÉ MARTIN}

École du Louvre 